\title{
Review
}

Magdalena Krintus* and Mauro Panteghini

\section{Laboratory-related issues in the measurement of cardiac troponins with highly sensitive assays}

https://doi.org/10.1515/cclm-2020-0017

Received January 8, 2020; accepted January 24, 2020

Abstract: A number of assay-related issues can affect the performance of cardiac troponin (cTn) measurement in everyday practice. In this respect, it is vital that all information on cTn assays is known and that the performance characteristics of assays are objectively assessed and adequately described. The advent of the latest generation of more sensitive cTn assays has heralded a new wave of information about low concentrations of cTn in blood. These recent generation assays have improved analytical sensitivity and corresponding performance at low cTn concentrations when compared to their predecessors, providing a convincing goal for laboratory medicine in helping clinicians in the diagnosis of acute myocardial infarction. Crucial to the clinical utility of highly sensitive cTn assays is the laboratorians' role in closely scrutinizing proposed assays and defining their value in relation to available evidence. Analytical, as well as pre-analytical and post-analytical, aspects must be documented. In this review, we describe what laboratory professionals should know about their cTn assay performance characteristics and the pre-analytical prerequisites for robustness to ensure optimal post-analytical reporting.

Keywords: cardiac troponin; highly sensitive assays; laboratory issues.

\section{Introduction}

The implementation of cardiac troponin (cTn) assays has played a groundbreaking role in the diagnosis, risk stratification and management of patients with suspected acute

*Corresponding author: Magdalena Krintus, Department of Laboratory Medicine, Nicolaus Copernicus University, Collegium Medicum, 9 Sklodowskiej-Curie Street, 85-094 Bydgoszcz, Poland, Phone: +485258544 90, Fax: +485258536 03,

E-mail: magdalena.krintus@wp.pl

Mauro Panteghini: Department of Biomedical and Clinical Sciences ‘Luigi Sacco', University of Milan, Milan, Italy myocardial infarction (AMI), stimulating a rapid evolution in cTn testing and concomitant innovations in clinical guidelines. Despite the performance of more highly sensitive cTn (hs-cTn) assays being infinitely superior to their predecessors, the issues relating to their introduction cannot be depreciated. In this regard, the role of medical laboratories is central as a number of assay-related issues can markedly affect the performance of cTn measurement in everyday practice [1]. Following the introduction of hs-cTn, it is therefore of vital importance to ensure that all analytical characteristics are specifically verified and that their performance is objectively assessed and adequately described. In this review, we discuss the fundamentals which laboratory professionals should know of hs-cTn assay performance characteristics and the pre-analytical prerequisites for robustness to ensure optimal postanalytical reporting.

\section{Using hs-cTn assays: a way of no return}

Due to their superior diagnostic accuracy, cTn tests have largely replaced other non-specific biomarkers, such as creatine kinase MB or myoglobin, for diagnosing suspected AMI [2]. The new millennium criteria for AMI and subsequent updates to the "universal definition of myocardial infarction" have strengthened the role of cTn as the preferred biomarker for the evaluation of AMI [3-5]. Afterward, the intensive efforts of in vitro diagnostics (IVD) manufacturers, aimed at improving assays for cTn measurement, resulted in the advent of hs-cTn assays, leading to the attendant laboratory scenario where several different assays are now available on the market. Paradoxically, the rapid evolution of assays has created an uncertainty among cardiologists regarding the clinical interpretation of cTn elevations [6]. Hence, many clinicians did not enthusiastically welcome more sensitive assays for $\mathrm{cTn}$ measurement and voiced concerns have appeared in clinical practice. The reaction of clinicians to hs-cTn assays is perfectly illustrated in the Robert Jesse's statement: "when troponin was a lousy assay it was a great test, but 
now that it's becoming a great assay, it's getting to be a lousy test" [7]. According to data from a survey carried out in 2016 involving 1900 medical centers in 23 countries evenly distributed across five continents, $41 \%$ of them adopted hs-cTn assays, varying from $60 \%$ in Europe to only $7 \%$ in North America [8]. More recently, the fourth universal definition of AMI, in providing practical updates to the previous 2012 version, has for the first time explicitly recommended the introduction of hs-cTn assays for their ability to detect even very small amounts of myocardial necrosis, expanding the use of cTn testing from the classical AMI diagnosis to the broad detection of myocardial injury of any cause [9]. This recommendation should theoretically have a global practice-changing impact, impelling medical laboratories to a rapid adoption of hs-cTn assays worldwide. The recent, as yet unpublished, update of the European Federation of Clinical Chemistry and Laboratory Medicine (EFLM) initiative on the Cardiac Marker Guideline Uptake in Europe (CARMAGUE) confirms this trend, as approximately two-thirds of 439 surveyed European laboratories declared to use cTn as a first-line test for suspected acute coronary syndrome (ACS), with most using hs-cTn assays (Päivi Laitinen, presented at the 23th IFCC-EFLM European Congress of Clinical Chemistry and Laboratory Medicine, May 2019).

Notably, measurement of cTn using highly sensitive assays reflects acknowledgment of scientific progress, and a universal acceptance of their use in diagnosing AMI appears inevitable. The difficulty lies in deciding how best to use this high-quality and previously unobtainable information. For sure, the introduction of hs-cTn assays requires changes to diagnostic rules and algorithms compelling laboratory professionals to ensure the safe clinical application of international recommendations on its use $[10,11]$. In contrast to a number of clinical guidance documents, there is still an insufficiency of laboratory practice recommendations on the use of hs-cTn assays. A special report recently issued jointly by a panel from the Academy of the American Association for Clinical Chemistry and the IFCC Task Force on the Clinical Application of Cardiac Bio-Markers constitutes substantial progress in this important but still neglected issue [11]. Among others, the group raises the issue of communication with clinicians and the importance of laboratory in educating clinicians about the influence of pre-analytical and analytical problems potentially confounding hs-cTn assay results [11]. To ensure optimal use of hs-cTn assays in clinical practice, the role of the laboratory is crucial indeed and should cover all aspects of the total examination process, from the pre-analytical to the post-analytical phase [12].

\section{The post-analytical phase: improving results' interpretation}

A special scrutiny concerns the post-analytical phase as an appropriate interpretation of hs-cTn results may directly influence major clinical decisions involving diagnosis, classification, monitoring and treatment of patients. The improved analytical sensitivity of hs-cTn assays has distinctly reinforced the evidence that the 99th percentile upper reference limit (URL), when applied as decision limit to a single result, is not more practically functional in the diagnosis of AMI [13]. The restricted utility of the 99th percentile URL has been demonstrated in a study by Ungerer et al. [14]. Of 150 contributory samples to the 99th percentile cut-off, only $20 \%$ were shared across three hs-cTn assays. These assays revealed pronounced disparities in samples with increased cTn concentrations, thereby precluding an agreement in clinical classification of patients in the evaluated cohort by using respective 99th percentile URLs [14]. Similar results were more recently published by Clerico et al. [15]. Studies on the biological variation of cTns have emphasized a very high individuality of these biomarkers, expressed as a low index of individuality, confirming major limitations for the use of population-based reference limits [16]. Accordingly, only serial testing in the same individual allows for the discrimination of pathophysiological mechanisms of cTn release [17]. Scrutiny of cTn release kinetics in blood is essential to differentiate acute from chronic myocardial damage and may help to understand the characteristics of underlying processes associated with cTn release [13]. Importantly, if we dichotomize typical/atypical cTn curves (based on 2-3 serial measurements) based on their ability to detect acute damage, a decisional criterion, related to the entity of cTn increase (i.e. the cTn delta change), should be defined to characterize cTn patterns indicative of acute myocardial necrosis [13]. However, though $70 \%$ of surveyed laboratories in Europe confirmed the correct use of cTn serial testing, the same number of laboratories stated that no criterion was used for the interpretation of biomarker's changes over time [18]. These observations highlight that many laboratories, which have introduced and currently employ hs-cTn assays, are not rigorous in the application of optimal approaches for the management of AMI [19-21]. The laboratory's attention should therefore better focus on how to facilitate the interpretation of hs-cTn results and correctly adhere to current clinical guidelines, for example, helping clinicians to understand significant hs-cTn changes in the same patient using the electronic laboratory reporting system for automated delta change calculation. 
Another undervalued but important issue is the correct use of measurement units in reporting cTn test results. A non-harmonized use of measurement units and unnecessary use of decimal numbers are potential sources of medical errors [22]. So, whenever possible, laboratory results should be presented as whole numbers. In avoiding the unnecessary use of decimals, which is a matter of patient safety, the use of ng/L as unit for cTn tests, which is also acceptable to the International System (SI) of measurement, is strongly recommended, regardless of the analytical sensitivity of the assay used [23]. Despite this, 43\% of laboratories surveyed by the CARMAGUE initiative are still using $\mu \mathrm{g} / \mathrm{L}$, followed by two or three decimal numbers.

\section{Optimizing analytical quality}

The introduction of hs-cTn assays has substantially increased the accuracy of AMI detection at presentation to the emergency department (ED). The sensitive quantification of cardiomyocyte injury has enabled the development and validation of rapid diagnostic algorithms, which substantially improves the early rule-out or rule-in strategies [24]. In general, in patients admitted to the ED with symptoms suspicious for ACS, the diagnosis of non-ST-segment elevation AMI (NSTEMI) can be ruled out immediately on presentation, with a negative predictive value of $>99.5 \%$ and a sensitivity of $>99 \%$, if the hs-cTn concentration is undetectable $[25,26]$. On the contrary, hs-cTn concentration multiples of the assay's 99th percentile URL support a diagnosis of NSTEMI with a high positive predictive value $(>85 \%)$, when the test results are evaluated in an appropriate clinical context $[27,28]$. For hs-cTn values ranging from detectable concentrations to multiples of the 99th percentile URL, decision can be made based on hs-cTn retesting at 1,2 or $3 \mathrm{~h}$ later, depending on the protocol used (Figure 1) [24].

In this clinical scenario, accurate calibration of hs-cTn assays in the low range of concentrations and a low analytical variation are of the utmost importance as even relatively small changes in assay performance in practice may significantly influence the proportion of patients who could be identified as suitable for discharge. It necessitates laboratories to engage in additional activities to ensure proper quality performance for hs-cTn measurements [11]. The tools that laboratories need to check performance at the low end of measuring range of hs-Tn assays are summarized in Table 1 [10, 29].

In general, calibration verification means the assaying of control materials of known concentration to substantiate the acceptability of the measuring system's alignment

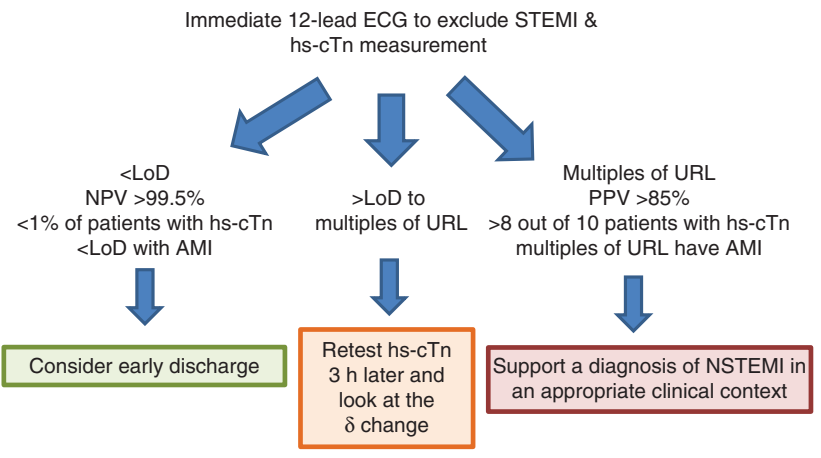

Figure 1: Decisional tree for optimal use of highly sensitive cardiac troponin assays in patients admitted to the emergency department with suspected acute myocardial infarction.

ECG, electrocardiogram; STEMI, ST-elevation myocardial infarction; hs-cTn, highly sensitive cardiac troponin assay; LoD, limit of detection of hs-cTn assay (i.e. the lowest amount of troponin in a biological sample that can be detected by the assay); NPV, negative predictive value; AMI, acute myocardial infarction; URL, upper reference limit defined at the 99th percentile of reference value distribution; PPV, positive predictive value; NSTEMI, non-STsegment elevation myocardial infarction.

Table 1: Recommended tools which medical laboratories should use to check the analytical performance at the low end of measuring range of their highly sensitive troponin (hs-cTn) assays.

\footnotetext{
- A control material or patient pool with an hs-cTn concentration near the assay limit of detection to monitor baseline drifts following assay calibration

- A low-level control material with a cTn concentration close to the 99th percentile upper reference limit to monitor assay variability at this decision level

- Calibration frequency to be determined based on the imprecision performance and drift characteristics of the assay
}

and, consequently, of patient results [30]. However, control materials offered by the manufacturers together with hs-cTn assays do not usually cover low cTn concentrations, leaving the assay vulnerable to potential drifts that may pass unnoticed. Aloisio et al. recently showed that daily monitoring of an in-house made serum pool with a concentration near the limit of detection (LoD) of the hs-cTn assay, used as an additional control material besides those offered by the manufacturer, improved the measurement accuracy of hs-cTn assays at low but clinically relevant concentrations [31]. Particularly, the laboratory results of the UK NEQAS Cardiac Markers External Quality Assessment (EQA) program, which includes a "low concentration sample" with cTn concentrations lower than the 99th percentile URL, were substantially better after the introduction of this additional quality control tool (the number of 
failed results in a 2-year period decreased from $40 \%$ to less than 4\%) [31]. Other authors also demonstrated that, when using a control material with hs-cTn concentrations close to the LoD, they were able to demonstrate significant differences (up to 100\%) in results between similar platforms from the same manufacturer [32]. On the contrary, no differences were seen using control materials with concentrations near to the assay's 99th percentile URL.

On the other hand, it is also important that laboratories evaluate the reproducibility of their hs-cTn assay at concentrations close to the 99th percentile URL to derive the measurement uncertainty at this decision level [33, 34]. This information should be obtained over a period (e.g. 6 consecutive months) sufficient to capture most changes to measuring conditions and systematic sources of uncertainty, such as those caused by different lots of reagents, different calibrations or different environmental conditions [35]. The characteristics of control material for estimating measurement uncertainty have been defined and should be carefully considered, i.e. the material should be different from that used to check the correct alignment of the measuring system, be commutable and with concentration corresponding to the decision cut-point employed in the medical application of the test [36]. The true test of how well measurement uncertainty holds up is therefore when hs-cTn assays are evaluated in daily practice and control materials closely resemble authentic patient samples [37]. HageSleiman et al. [38] have elegantly shown the misleading results obtained in the estimate of hs-cTn precision profile when using a non-commutable control material. The UK Association for Clinical Biochemistry and Laboratory Medicine has endorsed the use of a third-party internal quality control material at or near the 99th percentile URL to verify assay reproducibility. Despite this recommendation, only $7 \%$ of surveyed laboratories in the UK were running a quality control sample with these characteristics [39].

Talking about the analytical performance of hs-cTn assays poses the vital question as to the requisite degree of quality needed and to what extent measurement uncertainty is tolerable without jeopardizing patient safety [40]. The basic concept underlying this issue arose from the EFLM Strategic Conference held in Milan in 2014, which defined analytical performance specifications (APSs) according to different models [41, 42]. Notably, cTn should be considered as an analyte for which the model based on the effect of analytical performance on clinical outcomes should be applied in order to define APS [43]. This model should be used when the measurand has a central and well-defined role in decision-making for specific disease or a given clinical situation, and test results should be interpreted through established criteria, and applies to measurands for which results would strongly influence patient's outcome, e.g. in terms of permissible misclassification rates [43]. These measurand's characteristics perfectly applies to cTn and its use in ACS decision-making.

Sheehan et al. [44] first demonstrated the effect of analytical performance of cTn measurement on diagnostic misclassification. Performing duplicate cTn measurements, these authors calculated the frequency at which the result of the second replicate fell in a different diagnostic category according to a predefined cut-off, thus defining the percentage of misclassified patients with suspected AMI based on assay imprecision (assuming unbiased results). As expected, the frequency of misclassification rose together with the assay CV. This approach was fully transcribed into recommendations made in 2012 by the Australasian Association of Clinical Biochemists, where the imprecision goal was modulated for achieving a predefined acceptable rate of misclassification. Assuming as acceptable diagnostic misclassification rates of $1.8 \%, 1.0 \%$ and $0.5 \%$, the corresponding APSs for cTn imprecision (as CV) derived from this outcome-based model were $<13 \%,<10 \%$ and $<6 \%$, respectively [45]. More recently, Lyon et al. evaluated the combined influence of assay variability and bias on the number of false-positive and false-negative results for AMI diagnosis, using simulation models at hs-cTn 99th percentile URL [46]. A false-positive rate of approximately $1 \%$ was obtained when both bias and imprecision (as CV) of measurements were kept around 10\% [46]. Table 2 summarizes the current recommendations

Table 2: Analytical performance specifications (APSs) for troponin measurement using highly sensitive assays (hs-cTn) and recommended approaches to optimize their analytical quality.

\footnotetext{
- hs-cTn APS must be targeted at the concentration corresponding to the assay's 99th percentile upper reference limit.

- A measurement uncertainty $\leq 10 \%$ at this marker concentration represents the desirable goal. According to the classical Fraser's paradigm for deriving APS for random variability [47], the quality level can be modulated to minimum $[10 \%+1 / 210 \%=15 \%]$ and optimal $[1 / 210 \%=5 \%]$ goals.

- If a bias greater than $\pm 10 \%$ is detected in the quality control, a readjustment of the measuring system must be undertaken to correct it. If the bias remains, the manufacturer should be requested to take an immediate investigation and eventually fix the problem with a corrective action, e.g. a process of reassigning the manufacturer's calibrators for correcting the detected bias.
} 
about APS for hs-cTn measurements based on the available information from studies using the outcome-based model and related approaches to optimize their analytical quality. In addition, for result interpretation in EQA, when control materials are measured by participants in singlicate and results include effects of both bias and imprecision errors, a total error specification of maximum $\pm 22.5 \%$ is recommended [48].

\section{Understanding the impact of pre-analytical and analytical interferences}

It is well known that the sample matrix-related issues, which result from differences between serum and plasma, and the use of different anticoagulants may negatively affect the accuracy of cTn results and should be thoroughly investigated [49]. Plasma is usually the matrix of choice in order to ensure timeliness in patients with suspected AMI in ED. The use of plasma reduces the turnaround time (TAT) of sample treatment by eliminating clotting time and avoids potential problems associated with prolonged clotting time in patients receiving anticoagulation therapy or with coagulation abnormality (e.g. liver failure). The use of serum also has limitations associated with the possible presence of fibrin clots due to insufficient centrifugation of the tube or centrifugation prior to clot retraction.

Studies using previous generations of cTn assays already revealed significant differences in the 99th percentile URLs derived in cardio-healthy reference populations when both serum and heparin plasma were used [50]. When hs-cTn assays were introduced, similar investigations confirmed the frequent presence of some bias when comparing serum with plasma samples. Results from a multicenter analytical evaluation of Roche Diagnostics hs-cTn T assay showed a mean difference of $-4 \%$ in $\mathrm{cTn} T$ values when lithium heparin plasma was compared with serum [51]. The inter-sample bias was also an issue for Abbott Diagnostics hs-cTn I assay, because mean differences in hs-cTn I concentrations of $-5.7 \%$ and $17.4 \%$ were observed between serum and EDTA plasma and serum and lithium heparin plasma, respectively [52]. Both manufacturers in their package inserts list however as acceptable for hs-Tn determination both serum and plasma (heparin and EDTA), simply advising that the sample types are not interchangeable. Interestingly, they report as an acceptability criterion for sample validation a correlation slope ranging from 0.8 to 1.2 , corresponding to a systematic bias of $\pm 20 \%$ in results, well above the desirable APS recommended in Table 2 [53]. The analytical impact of the use of samples different from those used in the characterization of hs-cTn assays (e.g. serum for Roche hs-cTn T and heparin plasma for Abbott hs-cTn I) may be therefore noticeable and should require appropriate assay (re)validation and, more importantly, the redefinition of clinical thresholds specific for each employed sample. Incidentally, Katrukha et al., using a proteomic analysis, showed a consistent difference in the composition of cTn T forms in simultaneously collected serum and heparin plasma samples from the same patients with AMI [54]. While heparin plasma samples contained full-sized cTn $\mathrm{T}(\sim 35 \mathrm{kDa})$, in serum samples cTn T was present in a $29-\mathrm{kDa}$ form as a probable result of thrombin-mediated cleavage of $\mathrm{cTn} \mathrm{T}$ molecule occurring in vitro during the clotting process.

Apart from sample matrix-related differences, other pre-analytical and analytical interferents can lead to unreliable hs-cTn results. Hemolyzed samples, though very undesirable, are common in daily laboratory practice with a prevalence ranging from 3.3\% of all routine samples to more than $12 \%$ in samples coming from ED $[55,56]$. Significantly decreased results due to a moderate degree of hemolysis (free hemoglobin concentration $>1 \mathrm{~g} / \mathrm{L}$ ) have been reported using cTn T assay and, more recently, confirmed for hs-cTn T assays, potentially leading to unrecognized myocardial injury [57-59]. Some evidence suggests that intracellular proteases like cathepsin E, released from erythrocytes when a sample is hemolyzed, may degrade cTn T leading to a significant decrease in marker detection [60]. Laboratories should not only be aware of the possible influence of hemolysis, but also of the issues dealing with its accurate detection and reporting. Unfortunately, practices for identifying and rejecting hemolyzed specimens are highly variable in medical laboratories. According to a recent European survey, hemolysis is still identified using visual detection by $30 \%$ of laboratories and only $42 \%$ are using automated hemolysis index (HI) detection [61]. It is well demonstrated that visual examination of serum/plasma samples is neither accurate nor sensitive for detecting relatively low amount of hemolysis [62, 63]. By applying a risk management approach, Luksic et al. have elegantly shown that visual inspection for hemolysis detection increased the risk of reporting inaccurate cTn T results, potentially affecting clinical decisionmaking and patient outcome [64]. This emphasizes the need to introduce automated HI detection for accurately managing the possible impact of hemolysis by the automatic transmission of hemolysis degree to the laboratory 
information system and to harmonize hemolysis detection strategies in medical laboratories by overcoming visual inspection and arbitrary judgment of sample quality [65]. The analytical performance of HI determination and the reproducibility of results appear satisfactory, providing that employed platforms are reporting HI results as continuous values [66].

Even when using the automated approach for HI detection, laboratories differ significantly in their practices for dealing with hemolyzed samples in cTn result reporting. According to a UK survey, $40 \%$ of laboratories utilizing hs-cTn T assay removed all results automatically when free hemoglobin is $>1 \mathrm{~g} / \mathrm{L}$, whilst $10 \%$ reported annotated hs-cTn T results indicating possible interference [39]. Other authors have proposed tiered hemolysis thresholds suggesting a protocol for reporting or not hs-cTn T results dependent on marker concentrations [67]. For example, considering the significance of markedly elevated hs-cTn T concentrations at patient admission to the ED [27] and the inhibiting effect of hemolysis, values $>100 \mathrm{ng} / \mathrm{L}$ could be reported even if the HI value is $>100$ (i.e. the critical limit for interference), while for hs-cTn T concentrations $<100 \mathrm{ng} / \mathrm{L}$, the results should be deleted and a repeat sample requested.

In general, interference thresholds for hemolysis are defined as the greatest concentration of free hemoglobin in the sample that does not compromise the accuracy of cTn results. Manufacturers define internally their acceptance criteria when evaluating and validating these interference thresholds. Although the declared hemolysis limit for marketed hs-cTn I assays is usually very high (up to 4-5 $\mathrm{g} / \mathrm{L}$ of free hemoglobin), the employed acceptance criteria for interference bias are however as high as $\pm 10 \%$, which corresponds to the entire budget of allowable bias for hs-cTn measurements reported in Table 2 [68]. Consuming all the permissible bias in evaluating the hemolysis interference is therefore impractical and unsuitable in the context of the measurement quality. More correctly, the interference bias tolerated in the hemolysis interference studies should be a part of the total allowable bias and not exceeding a fraction of it [69].

As the LoD of hs-cTn assays is significantly lowered, it is necessary to carefully consider potential sources of non-specificity that have not been frequently detected in previous generations of less sensitive assays [70]. Inconsistent profiles of patient's test results, unexplained change in hs-cTn concentrations or disproportionate increases in measured values should suggest investigating interferences in suspected samples. Heterophile antibodies (HAs) remain the most frequently reported confounding factor giving both false-positive and false-negative hs-cTn results [71]. If an HA interference is suspected, the laboratories should implement appropriate strategies for detecting these interferents by: (a) checking linearity following serial dilutions, (b) reanalyzing samples using a different measuring system, or (c) measuring hs-cTn concentrations before and after the use of HA blocking reagents [29]. Although manufacturers have optimized their hs-cTn assays to reduce HA interferences by using chimeric human-mouse antibodies and by the addition of HA blocking agents to assay reagents, the occurrence of this interference has not been completely eliminated [72].

False-positive hs-cTn results may also be attributed to the presence of complexes between antibodies and cTn, resulting in a delayed clearance of the macrocomplex from the circulation. Initially recognized by Plebani et al. [73], the presence of macrotroponin I was identified in $5 \%$ of patients with elevated hs-cTn I values, when measured with the Abbott Architect assay; but given the biological characteristics of the interferent, the investigated assay may not be the only hs-cTn I assay affected by this problem [74]. A case of macrotroponin $\mathrm{T}$ has been also reported [75]. The presence of macrotroponin (I or T) in a suspected sample can be demonstrated by using the classical polyethylene glycol 6000 precipitation [76].

Biotin interference, potentially affecting immunoassays employing streptavidin-biotin binding technology, has been recently widely debated [77]. This assay architecture is used in $\sim 60 \%$ of marketed automated immunoassays (bioMérieux, Ortho Clinical Diagnostics, Roche Diagnostics, Siemens Healthcare Diagnostics, etc.) as the highly specific interaction of streptavidin with biotinylated antibodies aids in the detection and targeting of biological measurands [78]. In blood samples from individuals taking biotin supplements, free biotin molecules can compete with the ability of streptavidin-coated microparticles to bind the biotinylated antibodies that have captured the target analyte, leading to inaccurate measurements [79]. Multivitamin formulations (containing biotin in microgram amounts) do not elevate plasma biotin concentrations sufficiently to interfere with immunoassays. However, high-dose biotin supplements or novel treatments with very high biotin doses for patients with multiple sclerosis have increased concerns about biotin interference [80]. In streptavidin-biotin binding immunoassays using a sandwich format, such as hs-cTn assays, the presence of biotin may decrease the signal intensity producing falsely low results that may go undetected and lead to potentially serious clinical implications such as inappropriate patient discharge. However, biotin 
interference thresholds between different hs-cTn assays can vary substantially, from 2.5 to $10,000 \mathrm{ng} / \mathrm{mL}[68$, 80]. Frame et al. have experimentally shown a negative interference on hs-cTn T assay at biotin concentrations $>30 \mathrm{ng} / \mathrm{mL}$ (approximately 10× lower than the fourthgeneration cTn T assay), while the Abbott hs-cTn I assay was not affected by biotin concentrations in blood up to $2000 \mathrm{ng} / \mathrm{mL}$, due to a different assay design [81]. Although these findings require caution in considering the impact of biotin intake on some hs-cTn assays, they did not elucidate the real clinical impact of this issue. The magnitude of biotin interference is dependent on: (a) type of assay (design, sample volume), (b) time since last biotin intake (maximum impact at $1-3 \mathrm{~h}$ after ingestion), (c) biotin dose and (d) kidney function (delayed clearance in case of renal impairment). Studies quantifying biotin concentrations in the ED setting estimated at $0.4-0.5 \%$ the prevalence of patients admitted with vitamin levels $>30 \mathrm{ng} / \mathrm{mL}$ [82, 83]. Mumma et al. [84] carried out a simulation of the potential negative clinical impact of biotin interference on hs-cTn T in a population of 850 patients with suspected AMI (prevalence $15 \%)$. In this cohort, biotin concentrations exceeding $20 \mathrm{ng} / \mathrm{mL}$ were found in only one patient, with a prevalence of $0.7 \%$ at $\mathrm{ED}$ admission. Based on a $42 \%$ reduction of hs-cTn $\mathrm{T}$ recovery at $100 \mathrm{ng} / \mathrm{mL}$ biotin, the authors estimated the range between 19.0 and $45.2 \mathrm{ng} / \mathrm{L}$ as the hs-cTn T concentrations that could potentially lead to false-negative AMI prediction. As $25 \%$ of patients with AMI had results in this range, the likelihood of falsenegative results due to biotin interference was estimated to be $0.026 \%$. This suggests a low clinical impact, equating to less than three patients per 10,000 with AMI potentially being affected. More importantly, the manufacturer has recently worked by modifying the hs-cTn T assay to provide substantially higher tolerance to biotin interference, i.e. an hs-cTn T recovery $\geq 96 \%$ for biotin concentrations of $1250 \mathrm{ng} / \mathrm{mL}$ [85].

\section{TAT: an additional indicator of hs-cTn assay performance}

TAT is another important laboratory-related issue in hs-cTn measurements and their clinical use. For hs-cTn testing, a TAT $\leq 60 \mathrm{~min}$ from the time of receipt of blood tubes in the central laboratory to result reporting to clinical wards has been recommended [11]. Meeting appropriate TAT to ensure timeliness in reporting hs-cTn results is a prerequisite for the implementation of fast track algorithms currently recommended in clinical guidelines [86]. Several strategies to improve TAT have been undertaken, involving multidisciplinary teams acting from the pre-analytical to the post-analytical phase of the total examination process [87]. Decreasing TAT may require modifications of existing workflow, including the use of pneumatic tube transportation and computerized order entry management [88]. hs-cTn testing exploiting both these tools, combined with a dedicated path, may enable faster pre-analytical management of patient samples and the quicker availability of hs-cTn results. We demonstrated that this strategy may substantially decrease hs-cTn TAT and permit the achievement of the 60-min goal for almost all assayed samples [89, 90].

Point-of-care (POC) testing has been proposed as an alternative to meet patient care needs in situations when TAT requirements cannot be met with central laboratory systems [91]. However, a performance gap always historically existed between central laboratory systems and POC cTn testing in terms of analytical sensitivity [92-94]. More importantly, studies designed to compare POC technologies with central laboratory testing have shown that the lower clinical sensitivity of POC assays may result in a great number of misclassified patients [95]. Quite recently, novel highly sensitive technologies have been used to develop POC systems meeting hs-cTn assay criteria, providing promising perspectives in delivering accurate and quick hs-cTn results [96].

\section{Definition of "high-sensitivity" cTn assays: do we really need it?}

In 2015, an expert opinion conventionally designated as "high sensitivity" those cTn assays able to measure the biomarker at the 99th percentile URL concentration with a CV $\leq 10 \%$ and to detect results above the assay LoD in $\geq 50 \%$ of healthy individuals [97]. More recently, the second criterion became more stringent as the designated "high sensitive" assays should be able to measure cTn in at least $50 \%$ of healthy men and women separately [11]. This designation of cTn assays as being "high sensitive" has been however criticized because of substantial drawbacks [98]. By definition, the imprecision performance is strongly dependent upon the 99th percentile limit cTn concentration, and this may induce some authors to artificially inflate the 99th percentile value to obtain much favorable data owing to the relationship existing between the concentration of the analyte and the assay imprecision (i.e. the higher the concentration, the better the precision) 
[29, 99]. More generally, the aforementioned definition lacks consistency and is affected by too many factors (such as experimental definition of LoD, selection of reference population, etc.) [100, 101]. For these reasons, an approach using evidence-based clinical information instead of one based on the number of healthy subjects with cTn concentrations $>$ LoD should be preferable [10]. Overall, the need for a definition of "high sensitivity" cTn assays appears scientifically questionable. With the ongoing development of cTn assays with still greater analytical precision and lower detection limits, any absolute definition is probably rendered superfluous. Undeniably, cTn measurement is not a static science, but represents a dynamic laboratory and clinical activity, which may be affected by many preanalytical, analytical and post-analytical factors [102].

\section{Conclusions}

Five years ago, in this journal we used the "high-sensitivity" cTn as a paradigm to show how laboratory medicine acts as the science that underpins medicine [13]. Crucial to the application of this biomarker and its recent measurement development is the laboratorians' role in closely scrutinizing proposed assays and defining their clinical application in relation to available evidence. Therefore, education of laboratorians is as important as educating clinicians. For laboratory professionals, a good understanding of hs-cTn assay-related issues and the correct monitoring of its performance are crucial for appropriate use and interpretation of hs-cTn results. Relevant education, ongoing training and proper communication in the use of hs-cTn assays are essential in delivering efficient, high-quality laboratory service and promoting concomitant better patient care.

Author contributions: All the authors have accepted responsibility for the entire content of this submitted manuscript and approved submission.

Research funding: None declared.

Employment or leadership: None declared.

Institutional research grants: M. Krintus, Abbott Diagnostics; M. Panteghini, Abbott Diagnostics, Konica Minolta, IL Werfen, Roche Diagnostics.

Consultancy: M. Panteghini, Konica Minolta, Singulex. Speaker fees/travel expenses: M. Krintus, Abbott Diagnostics, Horiba Medical.

Platform loans/reagent gifts: M. Krintus, Abbott Diagnostics, Becton Dickinson; M. Panteghini, Abbott Diagnostics, The Binding Site, Brahms, Fujirebio, Greiner, Roche Diagnostics, Technopath.

\section{References}

1. Panteghini M. Performance of today's cardiac troponin assays and tomorrow's. Clin Chem 2002;48:809-10.

2. Kozinski M, Krintus M, Kubica J, Sypniewska G. High-sensitivity cardiac troponin assays: from improved analytical performance to enhanced risk stratification. Crit Rev Clin Lab Sci 2017;54:143-72.

3. The Joint European Society of Cardiology/American College of Cardiology Committee. Myocardial infarction redefined - a consensus document of the Joint European Society of Cardiology/ American College of Cardiology Committee for the redefinition of myocardial infarction. Eur Heart J 2000;21:1502-13.

4. Thygesen K, Alpert JS, White HD. Universal definition of myocardial infarction. Eur Heart J 2007;28:2525-38.

5. Thygesen K, Alpert JS, Jaffe AS, Simoons ML, Chaitman BR, White HD. Third universal definition of myocardial infarction. Eur Heart J 2012;33:2551-67.

6. Morrow DA. Clinical application of sensitive troponin assays. N Engl J Med 2009;361:913-5.

7. Jesse RL. On the relative value of an assay versus that of a test. A history of troponin for the diagnosis of myocardial infarction. J Am Coll Cardiol 2010;55:2125-8.

8. Anand A, Shah AS, Beshiri A, Jaffe AS, Mills NL. Global adoption of high-sensitivity cardiac troponins and the universal definition of myocardial infarction. Clin Chem 2019;65:484-9.

9. Thygesen K, Alpert JS, Jaffe AS, Chaitman BR, Bax JJ, Morrow DA, et al. Fourth universal definition of myocardial infarction (2018). Eur Heart J 2019;40:237-69.

10. Panteghini M. How clinical laboratories may improve their performance: the "high-sensitivity" troponin paradigm. Clin Chem 2018;64:621-3.

11. Wu AH, Christenson RH, Greene DN, Jaffe AS, Kavsak PA, Ordonez-Llanos J, et al. Clinical laboratory practice recommendations for the use of cardiac troponin in acute coronary syndrome: expert opinion from the Academy of the American Association for Clinical Chemistry and the Task Force on Clinical Applications of Cardiac Bio-Markers of the International Federation of Clinical Chemistry and Laboratory Medicine. Clin Chem 2018;64:645-55.

12. Plebani M. Total testing process: roots and state-of-the-art [published online ahead of print, 2019 Oct 1]. Diagnosis (Berl) 2020;7:19-20.

13. Ferraro S, Panteghini M. Laboratory medicine as the science that underpins medicine: the "high-sensitivity" troponin paradigm. Clin Chem Lab Med 2015;53:653-64.

14. Ungerer JP, Tate JR, Pretorius CJ. Discordance with 3 cardiac troponin I and T assays: implications for the 99th percentile cutoff. Clin Chem 2016;62:1106-14.

15. Clerico A, Ripoli A, Zaninotto M, Masotti S, Musetti V, Ciaccio M, et al. Head-to-head comparison of plasma cTnl concentration values measured with three high-sensitivity methods in a large Italian population of healthy volunteers and patients admitted to emergency department with acute coronary syndrome: a multi-center study. Clin Chim Acta 2019;496:25-34.

16. Wu AH, Lu QA, Todd J, Moecks J, Wians F. Short- and long-term biological variation in cardiac troponin I measured with a highsensitivity assay: implications for clinical practice. Clin Chem 2009;55:52-8. 
17. Neumann JT, Twerenbold R, Ojeda F, Sorensen NA, Chapman AR, Shah AS, et al. Application of high-sensitivity troponin in suspected myocardial infarction. N Engl J Med 2019;380:2529-40.

18. Collinson P, Hammerer-Lercher A, Suvisaari J, Apple FS, Christenson RH, Pulkki K, et al. How well do laboratories adhere to recommended clinical guidelines for the management of myocardial infarction: the cardiac marker guidelines uptake in Europe study (CARMAGUE). Clin Chem 2016;62:1264-71.

19. Thygesen K, Mair J, Giannitsis E, Mueller C, Lindahl B, Blankenberg $\mathrm{S}$, et al. How to use high-sensitivity cardiac troponins in acute cardiac care. Eur Heart J 2012;33:2252-7.

20. Hamm CW, Bassand JP, Agewall S, Bax J, Boersma E, Bueno H, et al. ESC Guidelines for the management of acute coronary syndromes in patients presenting without persistent ST-segment elevation. The Task Force for the management of acute coronary syndromes (ACS) in patients presenting without persistent ST-segment elevation of the European Society of Cardiology (ESC). Eur Heart J 2011;32:2999-3054.

21. Roffi M, Patrono C, Collet J-P, Mueller C, Valgimigli M, Andreotti F, et al. 2015 ESC Guidelines for the management of acute coronary syndromes in patients presenting without persistent ST-segment elevation: task force for the management of acute coronary syndromes in patients presenting without persistent ST-segment elevation of the European Society of Cardiology (ESC). Eur Heart J 2016;37:267-315.

22. Sinnott M, Eley R, Steinle V, Boyde M, Trenning L, Dimeski G. Decimal numbers and safe interpretation of clinical pathology results. J Clin Pathol 2014;67:179-81.

23. Barth JH, Panteghini M, Bunk DM, Christenson RH, Katrukha A, Noble JE, et al. Recommendation to harmonize the units for reporting cardiac troponin results. Clin Chim Acta 2014;432:166.

24. Twerenbold R, Boeddinghaus J, Nestelberger T, Wildi K, Rubini Gimenez M, Badertscher P, et al. How to best use high-sensitivity cardiac troponin in patients with suspected myocardial infarction. Clin Biochem 2018;53:143-55.

25. Body R, Mueller C, Giannitsis E, Christ M, Ordonez-Llanos J, de Filippi CR, et al. The use of very low concentrations of high-sensitivity troponin $\mathrm{T}$ to rule out acute myocardial infarction using a single blood test. Acad Emerg Med 2016;23:1004-13.

26. Carlton E, Greenslade J, Cullen L, Body R, Than M, Pickering JW, et al. Evaluation of high-sensitivity cardiac troponin I levels in patients with suspected acute coronary syndrome. JAMA Cardiol 2016;1:405-12.

27. Mueller-Hennessen M, Mueller C, Giannitsis E, Biener M, Vafaie $M$, deFilippi $C R$, et al. Serial sampling of high-sensitivity cardiac troponin T may not be required for prediction of acute myocardial infarction diagnosis in chest pain patients with highly abnormal concentrations at presentation. Clin Chem 2017;63:542-51.

28. Boeddinghaus J, Nestelberger T, Badertscher P, Twerenbold R, Fitze $B$, Wussler D, et al. Predicting acute myocardial infarction with a single blood draw. Clin Chem 2019;65:437-50.

29. Panteghini M. Assay-related issues in the measurement of cardiac troponins. Clin Chim Acta 2009;402:88-93.

30. Braga F, Infusino I, Panteghini M. Role and responsibilities of laboratory medicine specialists in the verification of metrological traceability of in vitro medical diagnostics. J Med Biochem 2015;34:282-7.

31. Aloisio E, Pasqualetti S, Dolci A, Panteghini M. Daily monitoring of a control material with a concentration near the limit of detection improves the measurement accuracy of highly sensitive troponin assays. Clin Chem Lab Med 2020;58:e29-31.

32. Kavsak PA, Don-Wauchope AC, Hill SA, Worster A. Acceptable analytical variation may exceed high-sensitivity cardiac troponin I cutoffs in early rule-out and rule-in acute myocardial infarction algorithms. Clin Chem 2016;62:887-9.

33. Braga F, Panteghini M. Defining permissible limits for the combined uncertainty budget in the implementation of metrological traceability. Clin Biochem 2018;57:7-11.

34. Ndreu R, Musetti V, Masotti S, Zaninotto M, Prontera C, Zucchelli $\mathrm{G}$, et al. Evaluation of reproducibility of the CTnT immunoassay using quality control samples. Clin Chim Acta 2019;495:269-70.

35. ISO/TS 20914:2019. Medical laboratories - Practical guidance for the estimation of measurement uncertainty, 1st ed. Geneva, Switzerland: ISO, 2019.

36. Braga F, Infusino I, Panteghini M. Performance criteria for combined uncertainty budget in the implementation of metrological traceability. Clin Chem Lab Med 2015;53:905-12.

37. Musetti V, Masotti S, Prontera C, Ndreu R, Zucchelli G, Passino $C$, et al. Evaluation of reference change values for a hs-cTnl immunoassay using both plasma samples of healthy subjects and patients and quality control samples. Clin Chem Lab Med 2019;57:e241-3.

38. Hage-Sleiman M, Capdevila L, Bailleul S, Lefevre G. Highsensitivity cardiac troponin-I analytical imprecisions evaluated by internal quality control or imprecision profile. Clin Chem Lab Med 2019;57:e49-51.

39. McKeeman GC, Auld PW. A national survey of troponin testing and recommendations for improved practice. Ann Clin Biochem 2015;52:527-42.

40. Panteghini M. Implementation of standardization in clinical practice: not always an easy task. Clin Chem Lab Med 2012;50:1237-41.

41. Sandberg S, Fraser CG, Horvath AR, Jansen R, Jones G, Oosterhuis W, et al. Defining analytical performance specifications: consensus statement from the 1st Strategic Conference of the European Federation of Clinical Chemistry and Laboratory Medicine. Clin Chem Lab Med 2015;53:833-5.

42. Panteghini M, Ceriotti F, Jones G, Oosterhuis W, Plebani M, Sandberg S. Strategies to define performance specifications in laboratory medicine: 3 years on from the Milan Strategic Conference. Clin Chem Lab Med 2017;55:1849-56.

43. Ceriotti F, Fernandez-Calle P, Klee GG, Nordin G, Sandberg S, Streichert T, et al. Criteria for assigning laboratory measurands to models for analytical performance specifications defined in the 1st EFLM Strategic Conference. Clin Chem Lab Med 2017;55:189-94.

44. Sheehan P, Blennerhassett J, Vasikaran SD. Decision limit for troponin I and assay performance. Ann Clin Biochem 2002;39:231-6.

45. Panteghini M. Quality requirements for troponin assays - an overview. In: Troponin monograph 2012, The Australasian Association of Clinical Biochemists Inc., 2012:53-61.

46. Lyon AW, Kavsak PA, Lyon OA, Worster A, Lyon ME. Simulation models of misclassification error for single thresholds of highsensitivity cardiac troponin I due to assay bias and imprecision. Clin Chem 2017;63:585-92.

47. Fraser CG, Hyltoft Petersen P, Libeer JC, Ricos C. Proposals for setting generally applicable quality goals solely based on biology. Ann Clin Biochem 1997;34:8-12. 
48. Tate JR, Panteghini M, Koerbin G, Hickman PE, Schneider HG, Jaffe AS. Verification of the analytical characteristics of troponin assays in the laboratory - a how to guide. In: Troponin monograph 2012, The Australasian Association of Clinical Biochemists Inc., 2012:69-85.

49. Jones GR, Panteghini M. Pre-analytical factors affecting troponin measurement. In: Troponin monograph 2012, The Australasian Association of Clinical Biochemists Inc., 2012:63-7.

50. Tate JR, Ferguson W, Bais R, Kostner K, Marwick T, Carter A. The determination of the 99th centile level for troponin assays in an Australian reference population. Ann Clin Biochem 2008;45:275-88.

51. Saenger AK, Beyrau R, Braun S, Cooray R, Dolci A, Freidank H, et al. Multicenter analytical evaluation of a high-sensitivity troponin T assay. Clin Chim Acta 2011;412:748-54.

52. Krintus M, Kozinski M, Boudry P, Capell NE, Köller U, Lackner K, et al. European multicenter analytical evaluation of the Abbott Architect STAT high sensitive troponin I immunoassay. Clin Chem Lab Med 2014;52:1657-65.

53. Pasqualetti S, Panteghini M. Highly sensitive troponin T measurement after pneumatic tube transportation: the sample type can make the difference. Clin Chim Acta 2019;S00098981(19):32078-9.

54. Katrukha IA, Kogan AE, Vylegzhanina AV, Serebryakova MV, Koshkina EV, Bereznikova AV, et al. Thrombin-mediated degradation of human cardiac troponin T. Clin Chem 2017;63:1094-100.

55. Lippi G, Plebani M, Di Somma S, Cervellin G. Hemolyzed specimens: a major challenge for emergency departments and clinical laboratories. Crit Rev Clin Lab Sci 2001;48:143-53.

56. Phelan MP, Reineks EZ, Schold JD, Hustey FM, Chamberlin J, Procop GW. Preanalytic factors associated with hemolysis in emergency department blood samples. Arch Pathol Lab Med 2018;142:229-35.

57. Lyon ME, Ball CL, Krause RD, Slotsve GA, Lyon AW. Effect of hemolysis on cardiac troponin T determination by the Elecsys 2010 immunoanalyzer. Clin Biochem 2004;37:698-701.

58. Li A, Brattsand G. Stability of serum samples and hemolysis interference on the high sensitivity troponin T assay. Clin Chem Lab Med 2011;49:335-6.

59. Trimboli F, Lucia F, Angotti E, Antico GC, Carinci Giacquinto L, Martucci M, et al. An approach based on simulated hemolysis for establishing the hemolysis index threshold for high-sensitivity cardiac troponin T assay. Clin Chem Lab Med 2019;57:e314-7.

60. Sodi R, Darn SM, Davison AS, Stott A, Shenkin A. Mechanism of interference by haemolysis in the cardiac troponin T immunoassay. Ann Clin Biochem 2006;43:49-56.

61. Cadamuro J, Lippi G, von Meyer A, Ibarz M, van Dongen-Lases $E$, Cornes $M$, et al. European survey on preanalytical sample handling - Part 2: Practices of European laboratories on monitoring and processing haemolytic, icteric and lipemic samples. On behalf of the European Federation of Clinical Chemistry and Laboratory Medicine (EFLM) Working Group for the Preanalytical Phase (WG-PRE). Biochem Med (Zagreb) 2019;29:020705.

62. Simundic AM, Nikolac N, Ivankovic V, Ferenec-Ruzic D, Magdic B, Kvaternik M, et al. Comparison of visual vs. automated detection of lipemic, icteric and hemolyzed specimens: can we rely on a human eye? Clin Chem Lab Med 2009;47:1361-5.

63. Lippi G, Cadamuro J. Visual assessment of sample quality: quo usque tandem? Clin Chem Lab Med 2018;56:513-5.
64. Luksic AH, Nikolac Gabaj N, Miler M, Dukic L, Bakliza A, Simundic AM. Visual assessment of hemolysis affects patient safety. Clin Chem Lab Med 2018;56:574-81.

65. Simundic AM, Baird G, Cadamuro J, Costelloe SJ, Lippi G. Managing hemolyzed samples in clinical laboratories. Crit Rev Clin Lab Sci 2020;57:1-21.

66. Aloisio E, Carnevale A, Pasqualetti S, Birindelli S, Dolci A, Panteghini M. Random uncertainty of photometric determination of hemolysis index on the Abbott Architect c16000 platform. Clin Biochem 2018;57:62-4.

67. Turner KA, Kaleta EJ, Harnish TL, Madani Z, Snozek CL. Evaluating tiered hemolysis thresholds to facilitate rapid reporting of high-sensitivity cardiac troponin. J Appl Lab Med 2019;4: 193-200.

68. Saenger AK, Jaffe AS, Body R, Collinson PO, Kavsak PA, Lam CS, et al. Cardiac troponin and natriuretic peptide analytical interferences from hemolysis and biotin: educational aids from the IFCC Committee on Cardiac Biomarkers (IFCC C-CB). Clin Chem Lab Med 2019;57:633-40.

69. Dolci A, Panteghini M. Harmonization of automated hemolysis index assessment and use: is it possible? Clin Chim Acta 2014;432:38-43.

70. Panteghini M. Avoid capture of interfering molecules in cardiac troponin immunoassays: working in shifts. Clin Biochem 2013;46:961-2.

71. Herman DS, Kavsak PA, Greene DN. Variability and error in cardiac troponin testing: an ACLPS critical review. Am J Clin Pathol 2017;148:281-95.

72. Mair J, Lindahl B, Müller C, Giannitsis E, Huber K, Möckel M, et al. What to do when you question cardiac troponin values. Eur Heart J Acute Cardiovasc Care 2018;7:577-86.

73. Plebani M, Mion M, Altinier S, Girotto MA, Baldo G, Zaninotto M. False-positive troponin I attributed to a macrocomplex. Clin Chem 2002;48:677-9.

74. Warner JV, Marshall GA. High incidence of macrotroponin I with a high-sensitivity troponin I assay. Clin Chem Lab Med 2016;54:1821-9.

75. Collinson PO, Mbedu M, Hunt C. Macrotroponin T causing a false positive troponin elevation. 70th AACC Annual Scientific Meeting Abstracts. Clin Chem 2018;64:S27.

76. Michielsen EC, Bisschops PG, Janssen MJ. False positive troponin result caused by a true macrotroponin. Clin Chem Lab Med 2011;49:923-5.

77. Update: The FDA warns that biotin may interfere with lab tests: FDA Safety Communication. Date issued: November 5, 2019. https://www.fda.gov/medical-devices/safety-communications/ update-fda-warns-biotin-may-interfere-lab-tests-fda-safetycommunication. Accessed: January 2020.

78. Biotin interference with troponin lab tests - assays subject to biotin interference. Date issued: May 11, 2019. https://www. fda.gov/medical-devices/vitro-diagnostics/biotin-interferencetroponin-lab-tests-assays-subject-biotin-interference. Accessed: January 2020.

79. Piketty ML, Polak M, Flechtner I, Gonzales-Briceño L, Souberbielle JC. False biochemical diagnosis of hyperthyroidism in streptavidin-biotin-based immunoassays: the problem of biotin intake and related interferences. Clin Chem Lab Med 2017;55:780-8.

80. Bowen R, Benavides R, Colón-Franco JM, Katzman BM, Muthukumar A, Sadrzadeh H, et al. Best practices in mitigating 
the risk of biotin interference with laboratory testing. Clin Biochem 2019;74:1-11.

81. Frame IJ, Joshi PH, Mwangi C, Gunsolus I, De Lemos JA, Das SR, et al. Susceptibility of cardiac troponin assays to biotin interference. Am J Clin Pathol 2019;151:486-93.

82. Katzman BM, Lueke AJ, Donato LJ, Jaffe AS, Baumann NA. Prevalence of biotin supplement usage in outpatients and plasma biotin concentrations in patients presenting to the emergency department. Clin Biochem 2018;60:11-6.

83. Trambas CM, Liu KC, Luu H, Louey W, Lynch C, Yen T, et al. Further assessment of the prevalence of biotin supplementation and its impact on risk. Clin Biochem 2019;65:64-5.

84. Mumma B, Diercks D, Ziegler A, Dinkel-Keuthage C, Tran N. Quantifying the prevalence of elevated biotin in a cohort with suspected acute coronary syndrome. 70th AACC Annual Scientific Meeting Abstracts. Clin Chem 2018;64:S35.

85. Imdahl R, Albert G, Kunzelmann S, Rank C, Zerback R, von Meyer. Performance evaluation of a new troponin T-high sensitive assay with increased tolerance to biotin. 71st AACC Annual Scientific Meeting Abstracts. Clin Chem 2019;65:S5.

86. Ferraro S, Dolci A, Panteghini M. Fast track protocols using highly sensitive troponin assays for ruling out and ruling in non-ST elevation acute coronary syndrome. Clin Chem Lab Med 2017;55:1683-9.

87. Boelstler AM, Rowland R, Theoret J, Takla RB, Szpunar S, Patel $\mathrm{SP}$, et al. Decreasing troponin turnaround time in the emergency department using the central laboratory: a process improvement study. Clin Biochem 2015;48:308-12.

88. Guss DA, Chan TC, Killeen JP. The impact of a pneumatic tube and computerized physician order management on laboratory turnaround time. Ann Emerg Med 2008;51:181-5.

89. Pasqualetti S, Birindelli S, Aloisio E, Dolci A, Panteghini M. Clinical governance remains a priority in total laboratory automation era. J Appl Lab Med 2019;4:130-2.

90. Dolci A, Giavarina D, Pasqualetti S, Szőke D, Panteghini M. Total laboratory automation: do stat tests still matter? Clin Biochem 2017;50:605-11.

91. Bingisser R, Cairns C, Christ M, Hausfater P, Lindahl B, Mair J, et al. Cardiac troponin: a critical review of the case for pointof-care testing in the ED. Am J Emerg Med 2012;30:1639-49.
92. Bruins Slot MH, van der Heijden GJ, Stelpstra SD, Hoes AW, Rutten FH. Point-of-care tests in suspected acute myocardial infarction: a systematic review. Int J Cardiol 2013;168:5355-62.

93. Wu AH. Recent advances in point-of-care diagnostics for cardiac markers. EJIFCC 2014;25:170-7.

94. Amundson BE, Apple FS. Cardiac troponin assays: a review of quantitative point-of-care devices and their efficacy in the diagnosis of myocardial infarction. Clin Chem Lab Med 2015;53:665-76.

95. Palamalai V, Murakami MM, Apple FS. Diagnostic performance of four point of care cardiac troponin I assays to rule in and rule out acute myocardial infarction. Clin Biochem 2013;46:1631-5.

96. Braga F, Aloisio E, Panzeri A, Nakagawa T, Panteghini M. Analytical validation of a highly sensitive point-of-care system for cardiac troponin I determination. Clin Chem Lab Med 2020;58:138-45.

97. Apple FS, Jaffe AS, Collinson P, Mockel M, Ordonez-Llanos J, Lindahl B, et al. IFCC educational materials on selected analytical and clinical applications of high sensitivity cardiac troponin assays. Clin Biochem 2015;48:201-3.

98. Lippi G. The mystifying nomenclature of cardiac troponin immunoassays. Scand J Clin Lab Invest 2014;74:273-7.

99. Panteghini M. A critical appraisal of experimental factors influencing the definition of the 99th percentile limit for cardiac troponins. Clin Chem Lab Med 2009;47:1179-82.

100. Clerico A, Zaninotto M, Ripoli A, Masotti S, Prontera C, Passino C, et al. The 99th percentile of reference population for CTnI and CTnT assay: methodology, pathophysiology and clinical implications. Clin Chem Lab Med 2017;55:1634-51.

101. Clerico A, Zaninotto M, Padoan A, Masotti S, Musetti V, Prontera $C$, et al. Evaluation of analytical performance of immunoassay methods for cTnl and cTnT: from theory to practice. Adv Clin Chem 2019;93:239-62.

102. Bodor GS. Cardiac troponins: molecules of many surprises. Clin Chem 2017;63:1059-60.

Article note: This paper is dedicated to the memory of Jillian R. Tate, a leader in the field of cardiac troponin science and an unforgettable friend. 